\title{
AIAA-2006-6144
}

\section{Practical Aspects of the Equation-Error Method for Aircraft Parameter Estimation}

\author{
Eugene A. Morelli \\ NASA Langley Research Center \\ Hampton, VA
}

\section{AIAA Atmospheric Flight Mechanics Conference August 21-24, 2006 / Keystone, CO}

For permission to copy or to republish, contact the American Institute of Aeronautics and Astronautics, 1801 Alexander Bell Drive. Suite 500. Reston. VA. 20191-4344 


\title{
Practical Aspects of the Equation-Error Method for Aircraft Parameter Estimation
}

\author{
Eugene A. Morelli ${ }^{*}$ \\ NASA Langley Research Center, Hampton, Virginia, 23681-2199, USA
}

\begin{abstract}
Various practical aspects of the equation-error approach to aircraft parameter estimation were examined. The analysis was based on simulated flight data from an F-16 nonlinear simulation, with realistic noise sequences added to the computed aircraft responses. This approach exposes issues related to the parameter estimation techniques and results, because the true parameter values are known for simulation data. The issues studied include differentiating noisy time series, maximum likelihood parameter estimation, biases in equation-error parameter estimates, accurate computation of estimated parameter error bounds, comparisons of equation-error parameter estimates with output-error parameter estimates, analyzing data from multiple maneuvers, data collinearity, and frequency-domain methods.
\end{abstract}

\section{Nomenclature}

$\begin{array}{ll}a_{x}, a_{y}, a_{z} & \text { body-axis translational accelerometer measurements, } \mathrm{ft} / \mathrm{sec}^{2} \\ b & \text { wing span, } \mathrm{ft} \\ \bar{c} & \text { mean aerodynamic chord, } \mathrm{ft} \\ C o v & \text { covariance matrix } \\ E\{\} & \text { expectation operator } \\ I_{x}, I_{y}, I_{z}, I_{x z} & \text { mass moments of inertia } \\ j & \text { imaginary number }=\sqrt{-1} \\ J & \text { cost function } \\ m & \text { mass, slugs } \\ p, q, r & \text { body-axis roll, pitch, and yaw rates, rad/sec } \\ \bar{q} & \text { dynamic pressure, lbf/ft } \\ R e(\quad) & \text { real part } \\ s & \text { standard error } \\ S & \text { wing reference area, ft } \\ T & \text { thrust, lbf } \\ V & \text { airspeed, ft/sec } \\ x_{c g}, y_{c g}, z_{c g} & \text { coordinates of the center of gravity } \\ x_{r e f}, y_{r e f}, z_{r e f} & \text { coordinates of the reference point } \\ \alpha & \text { angle of attack, rad } \\ \beta & \text { sideslip angle, rad } \\ \delta_{s}, \delta_{a}, \delta_{r} & \text { stabilator, aileron, and rudder deflections, deg } \\ \sigma^{2}, \text { Var } & \text { varameter vector } \\ & \end{array}$

\footnotetext{
${ }^{*}$ Research Engineer, Dynamic Systems and Control Branch, MS 308, Associate Fellow
} 


\section{superscripts}

$\begin{array}{ll}T & \text { transpose } \\ \sim & \text { estimate } \\ \cdot & \text { Fourier transform } \\ -1 & \text { time derivative } \\ + & \text { matrix inverse } \\ & \text { complex conjugate transpose }\end{array}$

subscripts

$O$

reference value

\section{Introduction}

The equation-error method was one of the first analytical techniques used to estimate aircraft dynamic model parameters from flight data ${ }^{1}$. In the 1960's and 1970's, the advent of the analog and digital computer, along with various theoretical developments and numerous practical successes made the output-error method ${ }^{2}$ the widely-accepted standard method for aircraft parameter estimation. However, recent analytical developments and special problems in aircraft dynamic modeling have highlighted the generality and usefulness of the equation-error approach. In this paper, several important practical aspects associated with using the equation-error method for aircraft parameter estimation are examined. In some cases, modern treatments of flight data negate earlier problems with using the equation-error method for flight data analysis. In other cases, the discussion shows that modern implementations of the equation-error method have important advantages for some aircraft modeling problems that have become prevalent.

Following a brief introduction to the equation-error method, the paper is arranged as a sequence of topics, each with analytical background and development, and numerical experiments using simulated data. The simulated data come mainly from a nonlinear F-16 simulation ${ }^{3}$. Noise sequences similar to those observed in real flight data analysis and modeling were added to the simulated aircraft responses. This approach makes it possible to identify practical problems and solutions regarding the parameter estimation, because the true parameter values are known from the simulation. All of the tools used to generate the results shown in the paper are available in a MATLAB ${ }^{\circledR 4}$

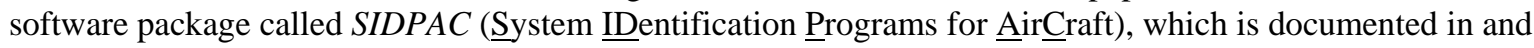
included with Ref. 5.

\section{Aircraft Parameter Estimation using Equation-Error}

In its most common form, the equation-error method calculates aerodynamic parameter estimates that minimize the sum of squared differences between values of non-dimensional force and moment coefficients determined from measured flight data and corresponding model values. The non-dimensional force and moment coefficients are computed by substituting measured and known quantities on the right sides of the following equations ${ }^{5}$

$$
\begin{gathered}
C_{X} \equiv-C_{A}=\frac{\left(m a_{x}-T\right)}{\bar{q} S} \quad C_{Y}=\frac{m a_{y}}{\bar{q} S} \quad C_{Z}=-C_{N}=\frac{m a_{z}}{\bar{q} S} \\
C_{D}=-C_{X} \cos \alpha-C_{Z} \sin \alpha \\
C_{l}=\frac{I_{X}}{\bar{q} S b}\left[\dot{p}-\frac{I_{x z}}{I_{x}}(p q+\dot{r})+\frac{\left(I_{z}-I_{y}\right)}{I_{X}} q r\right] \\
C_{m}=\frac{I_{y}}{\bar{q} S \bar{c}}\left[\dot{q}+\frac{\left(I_{x}-I_{z}\right)}{I_{y}} p r+\frac{I_{x z}}{I_{y}}\left(p^{2}-r^{2}\right)\right]
\end{gathered}
$$




$$
C_{n}=\frac{I_{z}}{\bar{q} S b}\left[\dot{r}-\frac{I_{x z}}{I_{z}}(\dot{p}-q r)+\frac{\left(I_{y}-I_{x}\right)}{I_{z}} p q\right]
$$

This produces $N$ values of the non-dimensional force and moment coefficients, where $N$ is the number of data points. These values are often called measured force and moment coefficients, even though they not measured directly, but rather computed from other measurements and known quantities.

Model values are computed from a postulated model for each force or moment coefficient in terms of measured aircraft states and controls. Although the form of the models can vary, typical linear model structures might be:

$$
\begin{gathered}
C_{L}=C_{L_{\alpha}} \alpha+C_{L_{\delta_{s}}} \delta_{s}+C_{L_{o}} \\
C_{D}=C_{D_{\alpha}} \alpha+C_{D_{\delta_{s}}} \delta_{s}+C_{D_{o}} \\
C_{m}=C_{m_{\alpha}} \alpha+C_{m_{q}} \frac{q \bar{c}}{2 V_{o}}+C_{m_{\delta_{s}}} \delta_{s}+C_{m_{o}} \\
C_{Y}=C_{Y_{\beta}} \beta+C_{Y_{\delta_{r}}} \delta_{r}+C_{Y_{o}} \\
C_{l}=C_{l_{\beta}} \beta+C_{l_{p}} \frac{p b}{2 V_{o}}+C_{l_{r}} \frac{r b}{2 V_{o}}+C_{l_{\delta_{a}}} \delta_{a}+C_{l_{\delta_{r}}} \delta_{r}+C_{l_{o}} \\
C_{n}=C_{n_{\beta}} \beta+C_{n_{p}} \frac{p b}{2 V_{o}}+C_{n_{r}} \frac{r b}{2 V_{o}}+C_{n_{\delta_{a}}} \delta_{a}+C_{n_{\delta_{r}}} \delta_{r}+C_{n_{o}}
\end{gathered}
$$

For each of the equations (6)-(11), substituting force or moment coefficient values computed from Eqs. (1)-(5) on the left side, along with corresponding measured states and controls on the right, results in an over-determined set of equations for the unknown aerodynamic parameters. This problem can be solved using a standard least-squares method.

For example, the least-squares problem for the pitching moment coefficient $C_{m}$ is formulated using the model structure in Eq. (8) as

$$
z=X \theta+v
$$

where

$$
\begin{gathered}
\boldsymbol{z}=\left[\begin{array}{cccc}
C_{m}(1) & C_{m}(2) & \ldots & C_{m}(N)
\end{array}\right]^{T}=N \times 1 \text { vector of values computed from Eq. (4) } \\
\boldsymbol{\theta}=\left[\begin{array}{llll}
C_{m_{\alpha}} & C_{m_{q}} & C_{m_{\delta_{s}}} & C_{m_{o}}
\end{array}\right]^{T}=4 \times 1 \text { vector of unknown parameters } \\
\boldsymbol{X}=\left[\begin{array}{llll}
\boldsymbol{\alpha} & \frac{q \bar{c}}{2 V_{o}} & \boldsymbol{\delta}_{s} & \mathbf{1}
\end{array}\right]=N \times 4 \text { matrix of explanatory data vectors or regressors } \\
\boldsymbol{v}=\left[\begin{array}{lllll}
v(1) & v(2) & \ldots & v(N)
\end{array}\right]^{T}=N \times 1 \text { vector of equation errors }
\end{gathered}
$$

The matrix $\boldsymbol{X}$ is assembled using measured data, with each column representing a modeling function, also called a regressor. The best estimator of $\boldsymbol{\theta}$ in a least-squares sense comes from minimizing the sum of squared differences between the dependent variable measurements $z$ and the model, 


$$
J(\boldsymbol{\theta})=\frac{1}{2}(\boldsymbol{z}-\boldsymbol{X} \boldsymbol{\theta})^{T}(\boldsymbol{z}-\boldsymbol{X} \boldsymbol{\theta})
$$

The least-squares solution for the unknown parameter vector $\boldsymbol{\theta}$ is ${ }^{5}$

$$
\hat{\boldsymbol{\theta}}=\left(\boldsymbol{X}^{T} \boldsymbol{X}\right)^{-1} \boldsymbol{X}^{T} \boldsymbol{z}
$$

The estimated parameter covariance matrix is computed from ${ }^{5}$

$$
\begin{gathered}
\operatorname{Cov}(\hat{\boldsymbol{\theta}}) \equiv E\left[(\hat{\boldsymbol{\theta}}-\boldsymbol{\theta})(\hat{\boldsymbol{\theta}}-\boldsymbol{\theta})^{T}\right]=\hat{\sigma}^{2}\left(\boldsymbol{X}^{T} \boldsymbol{X}\right)^{-1} \equiv\left[C_{i j}\right] \quad i, j=1,2, \ldots, n_{p} \\
\hat{\sigma}^{2}=\frac{(\boldsymbol{z}-\hat{\boldsymbol{y}})^{T}(\boldsymbol{z}-\hat{\boldsymbol{y}})}{\left(N-n_{p}\right)} \quad \hat{\boldsymbol{y}}=\boldsymbol{X} \hat{\boldsymbol{\theta}}
\end{gathered}
$$

where the number of unknown parameters $n_{p}=4$ for this example. The standard errors of the estimated parameters are given by the square root of the diagonal elements of the covariance matrix,

$$
s\left(\hat{\theta}_{j}\right)=\sqrt{C_{j j}} \quad j=1,2, \ldots, n_{p}
$$

There are two important unstated assumptions underlying the expressions given above. One is that the regressors, or columns of $\boldsymbol{X}$, are assumed to be known without error. The second is that the noise on the dependent variable $\boldsymbol{z}$ is assumed to be white and Gaussian. In practical aircraft problems, neither of these assumptions holds. The consequences of this are discussed below, along with practical ways to correct the problems.

To apply equation-error parameter estimation in the frequency domain, the data is first transformed from the time domain into the frequency domain. For parameter estimation purposes, this can be done very effectively using a high-accuracy chirp-z Fourier transform, with the capability to use arbitrary frequencies for the transformation ${ }^{5,6}$. Prior to the development of this technique, it was not practical to apply the equation-error method in the frequency domain, because the standard method for Fourier transformation of sampled data introduced errors in the transformed data and the spacing of the transform frequencies in the frequency band of interest was often coarse. For the present work, the frequencies used for all Fourier transformations were

$$
f=\left[\begin{array}{lllll}
0.20 & 0.24 & \ldots & 1.16 & 1.2
\end{array}\right]^{T} \mathrm{~Hz}
$$

In general, frequency resolution of $0.04 \mathrm{~Hz}$ works well. The lower bound should be chosen as $2 / T$, where $T$ is the time length of the maneuver, and the upper bound should be chosen to include the dynamics of interest, typically around $1.2 \mathrm{~Hz}$ for rigid-body modes of full-scale aircraft.

The resulting set of over-determined equations is of the same form given in Eq. (12) above, except that the number of data points is $m$, which is the number of selected frequencies for the Fourier transformation, and the problem now involves complex numbers. The analogs of Eqs. (14)-(17) for equation-error in the frequency domain $\operatorname{are}^{5}$

$$
\begin{gathered}
\hat{\boldsymbol{\theta}}=\left[\operatorname{Re}\left(\tilde{\boldsymbol{X}}^{\dagger} \tilde{\boldsymbol{X}}\right)\right]^{-1} \operatorname{Re}\left(\tilde{\boldsymbol{X}}^{\dagger} \tilde{\boldsymbol{z}}\right) \\
\operatorname{Cov}(\hat{\boldsymbol{\theta}})=\hat{\sigma}^{2}\left[\operatorname{Re}\left(\tilde{\boldsymbol{X}}^{\dagger} \tilde{\boldsymbol{X}}\right)\right]^{-1} \equiv\left[C_{i j}\right] \quad i, j=1,2, \ldots, n_{p}
\end{gathered}
$$




$$
\begin{gathered}
\hat{\sigma}^{2}=\frac{(\tilde{\boldsymbol{z}}-\hat{\tilde{\boldsymbol{y}}})^{\dagger}(\tilde{\boldsymbol{z}}-\hat{\tilde{\boldsymbol{y}}})}{m-n_{p}} \quad \hat{\tilde{\boldsymbol{y}}}=\tilde{\boldsymbol{X}} \hat{\boldsymbol{\theta}} \\
s\left(\hat{\theta}_{j}\right)=\sqrt{C_{j j}} \quad j=1,2, \ldots, n_{p}
\end{gathered}
$$

Note that Eqs. (19)-(22) are the same as Eqs. (14)-(17) when the data are real. Consequently, Eqs. (19)-(22) can be used for either real or complex data. When the data are complex, the expressions in Eqs. (19)-(22) are equivalent to using Eqs. (14)-(17) with the real and imaginary parts of the complex data arranged so that the imaginary values are stacked below the real parts, and treating the resulting problem as if the data were real. For example, if $\tilde{\boldsymbol{X}}=\boldsymbol{X}_{R}+j \boldsymbol{X}_{I}$, where $\boldsymbol{X}_{R}$ and $\boldsymbol{X}_{I}$ are $N \times n_{p}$ matrices of real numbers, then

$$
\operatorname{Re}\left(\tilde{\boldsymbol{X}}^{\dagger} \tilde{\boldsymbol{X}}\right)=\operatorname{Re}\left[\left(\boldsymbol{X}_{R}-j \boldsymbol{X}_{I}\right)^{T}\left(\boldsymbol{X}_{R}+j \boldsymbol{X}_{I}\right)\right]=\boldsymbol{X}_{R}^{T} \boldsymbol{X}_{R}+\boldsymbol{X}_{I}^{T} \boldsymbol{X}_{I}=\left[\begin{array}{c}
\boldsymbol{X}_{R} \\
\boldsymbol{X}_{I}
\end{array}\right]^{T}\left[\begin{array}{c}
\boldsymbol{X}_{R} \\
\boldsymbol{X}_{I}
\end{array}\right]
$$

and similarly for $\operatorname{Re}\left(\tilde{\boldsymbol{X}}^{\dagger} \tilde{\boldsymbol{z}}\right)$. It follows that the regression problem for complex data can be treated as a problem with real data by simply stacking the real and imaginary parts of the complex data. However, the value of $N$ in Eq. (16) must equal the number of data points, which will be the number of frequency points $m$.

The equations above show that the equation-error method for parameter estimation has a relatively simple, non-iterative solution, based on linear algebra. The modeling can be done using any of the Eqs. (6)-(11) (or analogous versions of these equations, with different model structures), one at a time. The equation-error method can also be applied to state space models to estimate dimensional stability and control derivatives, see Ref. 5 . The equation-error method can be used very efficiently for very large data sets, e.g, from wind tunnel tests or multiple flight test maneuvers. This efficiency is also needed when identifying nonlinear models, which typically involves evaluating many different candidate model structures by estimating the parameters for each candidate model structure and using statistical modeling metrics to choose among them. The efficiency of the equation-error method makes it the only practical choice for this problem.

The equation-error method can be considered a method wherein the model matches state time-derivative information from the dynamic system, rather than matching the states or outputs, as in the output-error method. This can be seen from the right sides of Eqs. (1)-(5), where the quantities used as dependent variables in the equationerror method are computed from translational and angular accelerations. Consequently, a model that uses equationerror parameter estimates will not produce the best match to the measured outputs of the dynamic system, since that is not what is being optimized. On the other hand, there is no need to integrate equations of motion to get model outputs when using the equation-error method, because the matching is done in the equations of motion themselves (hence the name "equation-error"). One important practical consequence is that the equation-error method can be applied equally well to data from inherently unstable aircraft flying under closed-loop feedback control.

\section{Practical Issues}

\section{A. Differentiating Noisy Time Series}

Eqs. (3)-(5) show that angular accelerations $\dot{p}, \dot{q}, \dot{r}$, are required to compute the measured values of the moment coefficients $C_{l}, C_{m}, C_{n}$. Usually, angular accelerations are either not measured or else the measurements are of poor quality. Consequently, angular accelerations must be determined by numerically differentiating the measured angular rates, which are noisy. Signal distortion of any kind can degrade modeling results, so it is important that this data processing step be done accurately and without introducing additional noise or signal distortion. There are many different methods for numerically differentiating noisy data, particularly in the biomedical field ${ }^{7}$. Methods that have been found to work well for aircraft data are described here. 
The main problem with differentiating noisy data is that straightforward finite difference formulas cannot be used, because noise on the data causes unacceptable distortions to the relatively small values computed for the numerator and denominator in a typical finite difference formula. Consequently, the data processing must be a combination of smoothing and differentiation.

One method is to sequentially fit local approximating polynomial models to the data, then differentiate the approximating polynomial. For example, 5 local points (two on either side of the data point where the derivative is to be computed) can be fit with a second order polynomial in time,

$$
y=a t^{2}+b t+c
$$

where $t$ is defined as zero at the center point and the constants $a, b$, and $c$ are estimated using least squares, as in Eq. (14). This corresponds to assuming that the acceleration is constant for these five consecutive data points. Then the derivative at the center point is computed as the derivative of the local approximating polynomial evaluated at the center point where $t \equiv 0$, which equals $b$. This approach can be moved along the time line, and modified for the endpoints, where there are fewer than 2 data points on one side of the point in question. References 5 and 8 explain this method, and provide practical algorithms for carrying it out. However, in practical cases, the degree of smoothing from this approach is often not sufficient, and additional data smoothing must be applied. This can be done with zero distortion and zero phase shift using a method based on Fourier analysis and the Wiener filter, called an optimal Fourier smoother, which is described in Refs. 5 and 9. The optimal Fourier smoother is a global method, because the technique is applied to all of the data from the maneuver at once, rather than operating sequentially on local subsets of the data.

Alternatively, the data can be smoothed initially using the optimal Fourier smoother, then differentiated using either the local polynomial method described earlier, or by computing the derivative of the smoothed signal in the frequency domain, based on the results from the optimal Fourier smoother. In summary, the three methods are:

1) Optimal Fourier smoother followed by the local polynomial smoothing derivative

2) Differentiating the smoothed signal from the optimal Fourier smoother in the frequency domain, then reconstructing the smoothed derivative in the time domain

3) Local polynomial smoothing derivative followed by the optimal Fourier smoother

To evaluate these methods, random colored noise sequences were used. The objective is to differentiate the band-limited portion of the signal, which simulates an arbitrary aircraft response, while rejecting the wide-band noise. This situation is very close to practical aircraft cases, and covers many possibilities by using random noise sequences to generate the band-limited part of the signal that simulates the aircraft motion. The band-limited part of the signal is known, and its derivative can be computed very accurately using numerical means. The numerical differentiation methods to be evaluated operate only on the noisy data, which include both the simulated deterministic part and the wide-band noise.

Figure 1 shows results from applying the three methods to 100 different colored noise sequences. Methods 1 and 2 gave similar results, with root-mean-square errors averaging around 5 percent. Method 3 was less accurate, because differentiating first (before optimal Fourier smoothing) raised the noise level somewhat, and this occasionally led to increased errors. By using the optimal Fourier smoother first, the noise was mostly removed, and the

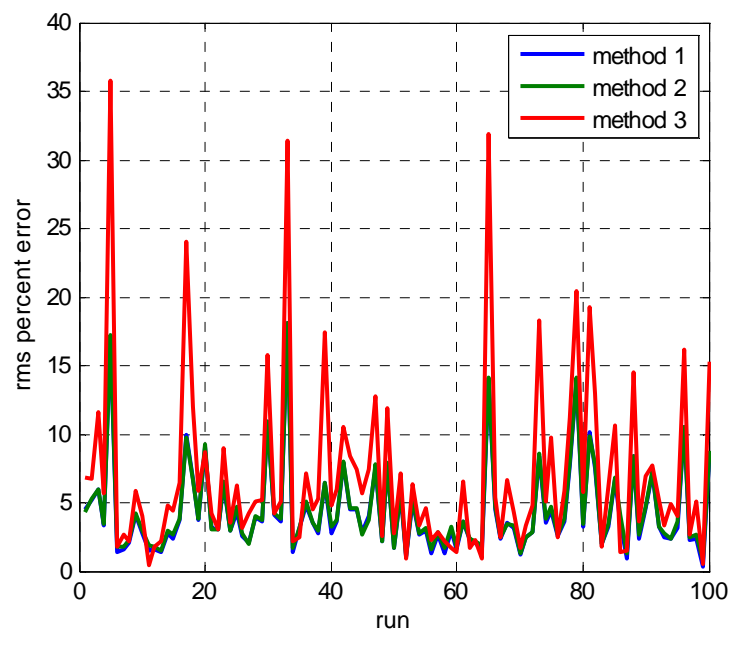

Figure 1. Root-mean-square error for numerical differentiation of noisy time series 
subsequent smooth numerical differentiation was more accurate. Figure 2 shows typical results using all three methods on a noisy time series. All three methods approximate the true derivative well, with relatively small differences between the true derivative and the numerical derivatives, shown in the plots on the right.
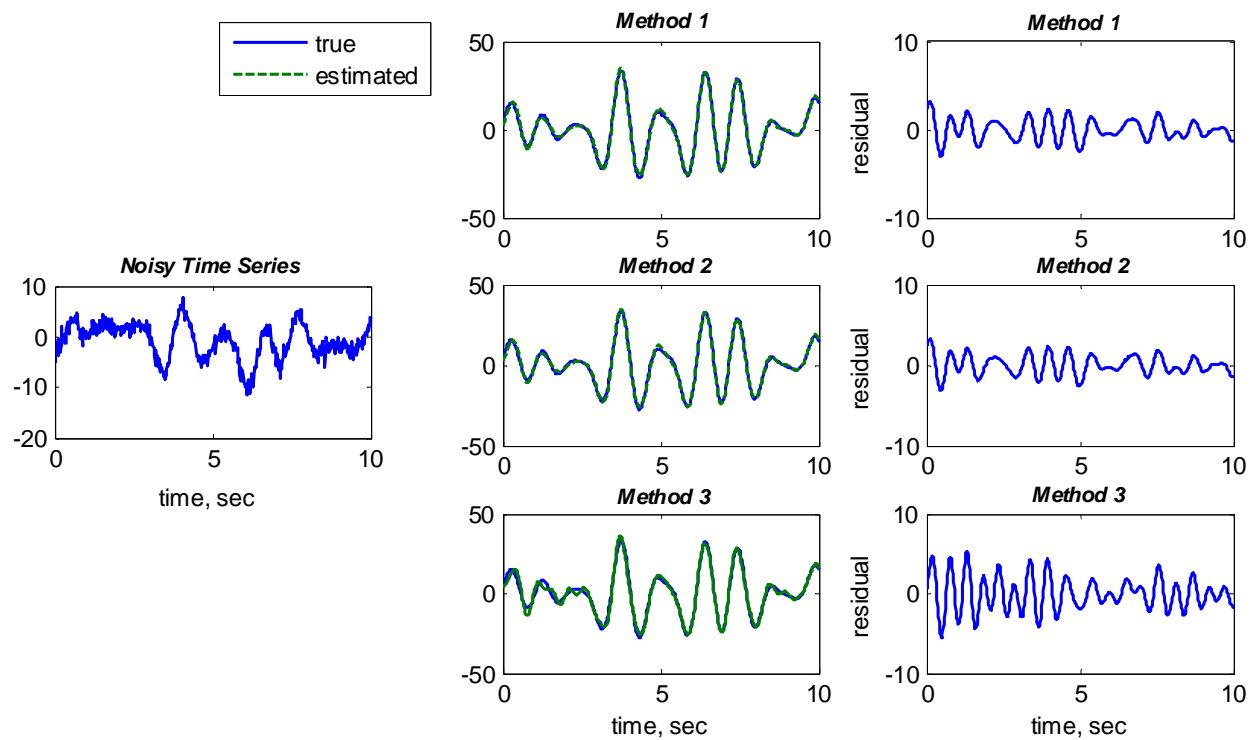

Figure 2. Typical example of numerical derivatives of noisy time series

All of the methods lose accuracy near the endpoints of the time series, but this problem can be eliminated by including an extra $1 \mathrm{sec}$ of data on either end and discarding the results for the extra data. This was done for all of the results shown in Figs. 1 and 2. The recommendation of $1 \mathrm{sec}$ on either end worked well for $50 \mathrm{~Hz}$ data with deterministic signal in the range of $[0,1] \mathrm{Hz}$. These frequencies are typical for practical aircraft problems. The time length for an effective endpoint buffer could be different for other cases.

The optimal Fourier smoother automatically computes a cut-off frequency for the smoother based on the data. This cut-off frequency can also be chosen manually by the analyst, based on plots of the Fourier coefficients. The best results were obtained when the cut-off frequency was about $0.5 \mathrm{~Hz}$ above the frequency band of the deterministic signal. This can be easily found visually, based on plots of the Fourier coefficient magnitudes produced in the optimal Fourier smoothing method. Again, this recommendation was based on $50 \mathrm{~Hz}$ data with a deterministic signal bandwidth of $[0,1] \mathrm{Hz}$. All of the results shown in Figs. 1 and 2 used automatic determination of the cut-off frequency, based on the data.

\section{B. Maximum Likelihood Estimates}

It is sometimes argued that output-error parameter estimates are superior to equation-error parameter estimates because output-error parameter estimates maximize a likelihood function, which is a statistical measure of conditional probability. Since the output-error parameter estimates have this theoretical underpinning, they are considered to be more valid and/or more accurate. In reality, either equation-error or output-error parameter estimates can be considered maximum likelihood estimates. The difference between the two approaches is in which aspect of the physical system response is being matched by the model, and in the assumptions about the noise. Consequently, the true distinction between the output-error and equation-error methods is in which assumptions are most useful and valid in modeling the dynamic response of the physical system.

The basis of maximum likelihood parameter estimation is the likelihood function, which is the conditional probability of the measurements given the parameters, assuming Gaussian noise on the measured $n_{o}$ outputs ${ }^{5}$, 


$$
\mathbb{L}(\boldsymbol{Z} ; \boldsymbol{\theta})=\left[(2 \pi)^{n_{o}}|\boldsymbol{R}|\right]^{-\frac{N}{2}} \exp \left\{-\frac{1}{2} \sum_{i=1}^{N}\left[\boldsymbol{z}_{i}-\boldsymbol{y}_{i}(\boldsymbol{\theta})\right]^{T} \boldsymbol{R}^{-1}\left[\boldsymbol{z}_{i}-\boldsymbol{y}_{i}(\boldsymbol{\theta})\right]\right\}
$$

where $\boldsymbol{z}_{i}$ is the $i^{\text {th }}$ measured output vector, $\boldsymbol{Z}$ represents all the $\boldsymbol{z}_{i}, \boldsymbol{y}_{i}$ is the $i^{\text {th }}$ model output vector that depends on the parameter vector $\boldsymbol{\theta}$, and $\boldsymbol{R}$ is the measurement noise covariance matrix. For simplicity, assume that there is only a single measured output (this is the typical case for the equation-error method), so that $\boldsymbol{Z}$ consists of a vector of $N$ measurements taken over the course of a maneuver, and $\boldsymbol{R}$ is a scalar. The maximum likelihood parameter estimate is obtained as the value of $\boldsymbol{\theta}$ that maximizes the likelihood function, which is equivalent to minimizing the negative log-likelihood function,

$$
\hat{\boldsymbol{\theta}}=\max _{\boldsymbol{\theta}} \mathbb{L}(\boldsymbol{Z} ; \boldsymbol{\theta})=\min _{\boldsymbol{\theta}}\{-\ln [\mathbb{L}(\boldsymbol{Z} ; \boldsymbol{\theta})]\}=\min _{\boldsymbol{\theta}}\left\{\frac{1}{2} \sum_{i=1}^{N}\left[\boldsymbol{z}_{i}-\boldsymbol{y}_{i}(\boldsymbol{\theta})\right]^{T} \boldsymbol{R}^{-1}\left[\boldsymbol{z}_{i}-\boldsymbol{y}_{i}(\boldsymbol{\theta})\right]+\frac{N}{2} \ln |\boldsymbol{R}|\right\}
$$

where the constant term was dropped because it has no effect on the optimization. For a scalar measurement,

$$
\hat{\boldsymbol{\theta}}=\min _{\boldsymbol{\theta}}\left\{\frac{1}{2 \sigma^{2}} \sum_{i=1}^{N}\left[z_{i}-y_{i}(\boldsymbol{\theta})\right]^{2}+\frac{N}{2} \ln \sigma^{2}\right\}
$$

where $\boldsymbol{R}=\sigma^{2}$ is the noise variance for the single measurement.

In the most commonly-used forms, both equation-error parameter estimation and output-error parameter estimation are based on minimizing the negative log-likelihood function. The difference is only in the definition of the output, and consequently where the noise appears. Assuming the output is an aircraft response, such as pitch rate, means that the noise (assumed to be on the model output) appears on the pitch rate measurement. This is the output-error approach, which is equivalent to assuming that the equations of motion are deterministic (no process noise), with measurement noise on the aircraft response variables. If instead the output is assumed to be non-dimensional pitching moment coefficient, the noise (still assumed to be on the model output) appears on the non-dimensional pitching moment coefficient. This is the equation-error approach, which is equivalent to assuming that the equations of motion include process noise, but there is no measurement noise on aircraft states and controls used to form the regressors. Both equation-error and output-error maximize a likelihood function and are therefore maximum likelihood parameter estimation techniques. It follows that the theoretical properties ${ }^{5}$ of the parameter estimates are the same, assuming that the underlying assumptions are valid. The real issue is which set of assumptions is most appropriate for the problem, how close reality is to those assumptions, and which aspect of the physical system dynamics should be matched by the model, i.e., what should be defined as the measured output to be matched by the model. Similar arguments apply for the case of multiple measured outputs.

A general technique, commonly called filter-error, can be used to find maximum likelihood parameter estimates assuming both process noise and measurement noise. However, there are computational and practical problems with this approach that have limited its use in practice, see Ref. 5.

In both the equation-error and output-error methods, the noise covariance matrix $\boldsymbol{R}$ is found using a relaxation technique, where $\boldsymbol{\theta}$ and $\boldsymbol{R}$ are optimized alternately while the other is held fixed, and the process is repeated until estimates of both $\boldsymbol{\theta}$ and $\boldsymbol{R}$ converge. The equation-error method is normally applied to one force or moment coefficient (i.e., one equation) at a time, in which case the relaxation technique is not necessary, because the solution from Eq. (27) does not change for a single measured output, regardless of the constant weighting $\sigma^{2}$. However, the estimate $\hat{\sigma}^{2}$ is necessary for calculation of the estimated parameter covariance matrix, cf. Eq. (16).

For the single measurement case in Eq. (27) where $\boldsymbol{R}=\sigma^{2}$, the maximum likelihood estimate of $\sigma^{2}$ is found by setting the partial derivative of the negative log-likelihood function with respect to $\sigma^{2}$ equal to zero, and solving for $\sigma^{2}$, 


$$
\hat{\sigma}^{2}=\frac{1}{N} \sum_{i=1}^{N}\left[z_{i}-y_{i}(\boldsymbol{\theta})\right]^{2}
$$

Usually, equation-error methods use $N-n_{p}$ rather than $N$ in the denominator above (cf. Eq. (16)), which theoretically produces an unbiased estimate of the noise variance, see Ref. 10. For values of $N$ and $n_{p}$ typical of flight test maneuvers, $N>>n_{p}$, so this change makes virtually no difference in practice. Similar operations for the multiple measurement case give

$$
\hat{\boldsymbol{R}}=\frac{1}{N} \sum_{i=1}^{N}\left[\boldsymbol{z}_{i}-\boldsymbol{y}_{i}(\boldsymbol{\theta})\right]\left[\boldsymbol{z}_{i}-\boldsymbol{y}_{i}(\boldsymbol{\theta})\right]^{T}
$$

If $\hat{\sigma}^{2}$ or $\hat{\boldsymbol{R}}$ are not computed from Eq. (28) or (29), then the resulting parameter estimates are not maximum likelihood estimates. The practical consequence of this is usually that the estimated parameter standard errors are not accurate.

\section{Biased Parameter Estimates}

When the model structure includes either too many terms or too few terms relative to the information content in the data, the resulting parameter estimates will be biased. This is true regardless of the parameter estimation method employed. In the equation-error method, the fact that the regressors are noisy (because they are assembled from measured data) also leads to biases in the parameter estimates. This can be demonstrated by assuming the matrix of regressors $\boldsymbol{X}$ is comprised of the true (noise-free) regressors $\boldsymbol{X}_{t}$, plus a zero-mean random noise vector $\boldsymbol{\varepsilon}$,

$$
\boldsymbol{X}=\boldsymbol{X}_{t}+\boldsymbol{\varepsilon}
$$

From Eqs. (12) and (14), the expected value of the estimated parameter vector $\hat{\boldsymbol{\theta}}$ is computed as

$$
E(\hat{\boldsymbol{\theta}})=E\left[\left(\boldsymbol{X}^{T} \boldsymbol{X}\right)^{-1} \boldsymbol{X}^{T}\left(\boldsymbol{X}_{t} \boldsymbol{\theta}+\boldsymbol{v}\right)\right]
$$

Assuming $\boldsymbol{v}$ and $\boldsymbol{\varepsilon}$ are uncorrelated with zero mean values, then $\boldsymbol{X}=\boldsymbol{X}_{t}+\boldsymbol{\varepsilon}$ and $\boldsymbol{v}$ are also uncorrelated, so that

$$
\begin{gathered}
E(\hat{\boldsymbol{\theta}})=E\left[\left(\boldsymbol{X}^{T} \boldsymbol{X}\right)^{-1} \boldsymbol{X}^{T} \boldsymbol{X}_{t} \boldsymbol{\theta}\right] \\
E(\hat{\boldsymbol{\theta}})=E\left[\left(\boldsymbol{X}^{T} \boldsymbol{X}\right)^{-1} \boldsymbol{X}^{T}(\boldsymbol{X}-\boldsymbol{\varepsilon}) \boldsymbol{\theta}\right] \\
E(\hat{\boldsymbol{\theta}})=\boldsymbol{\theta}-E\left[\left(\boldsymbol{X}^{T} \boldsymbol{X}\right)^{-1} \boldsymbol{X}^{T} \boldsymbol{\varepsilon} \boldsymbol{\theta}\right]
\end{gathered}
$$

The parameter estimate $\hat{\boldsymbol{\theta}}$ computed using noisy regressors is biased by the quantity $-E\left[\left(\boldsymbol{X}^{T} \boldsymbol{X}\right)^{-1} \boldsymbol{X}^{T} \boldsymbol{\varepsilon} \boldsymbol{\theta}\right]$.

An F-16 simulation was used to investigate the link between noise on the regressors and bias in the estimated parameters using the equation-error method. Geometry and mass properties of the F-16 are given in Table 1 . To isolate the effects of regressor noise, a linear model for the F-16 short period dynamics was extracted from the 
nonlinear simulation at a flight condition of 5 deg trim angle of attack and 10,000 ft altitude, using central finite differences. The form of the linear model is:

$$
\begin{gathered}
\dot{\alpha}=Z_{\alpha} \alpha+\left(1+Z_{q}\right) q+Z_{\delta_{s}} \delta_{s} \\
\dot{q}=M_{\alpha} \alpha+M_{q} q+M_{\delta_{s}} \delta_{s} \\
a_{z}=\frac{V_{o}}{g}\left(Z_{\alpha} \alpha+Z_{q} q+Z_{\delta_{s}} \delta_{s}\right)
\end{gathered}
$$

where

$$
\begin{array}{ccc}
Z_{\alpha}=\frac{\bar{q}_{o} S}{m V_{o}} C_{Z_{\alpha}} & Z_{q}=\frac{\bar{q}_{o} S \bar{c}}{2 m V_{o}^{2}} C_{Z_{q}} & Z_{\delta_{s}}=\frac{\bar{q}_{o} S}{m V_{o}} C_{Z_{\delta_{s}}} \\
M_{\alpha}=\frac{\bar{q}_{o} S \bar{c}}{I_{y}} C_{m_{\alpha}} & M_{q}=\frac{\bar{q}_{o} S \bar{c}^{2}}{2 V_{o} I_{y}} C_{m_{q}} & M_{\delta_{s}}=\frac{\bar{q}_{o} S \bar{c}}{I_{y}} C_{m_{\delta_{s}}}
\end{array}
$$

This linear model was used to generate simulated data, to avoid any bias in the parameter estimates from model structure errors, and thereby isolate the effects of regressor noise on estimated parameter bias. The stabilator input was a sequence of square wave pulses with pulse widths in the ratio 2-1-1-2, where the 1 pulse had a width of 1 sec, and the amplitude of the input was $1 \mathrm{deg}$. Other square waves and frequency sweep inputs with various input amplitudes were also used, with similar results to those given next.

Several methods were used to estimate the non-dimensional model parameters:

1) Equation-error least squares using noise-free regressors

2) Equation-error least squares using noisy regressors

3) Equation-error least squares in the frequency domain using noisy regressors

4) Equation-error least squares with noisy regressors smoothed using optimal Fourier smoothing

5) Output-error in the time domain with noisy outputs

Repeated maneuvers were run using the same input and simulation, but different noise sequences, and all of the methods listed above were applied to estimate the model parameters from the simulated data. Figure 3 shows a summary of the results. Only the bias is shown for each estimated parameter, which was computed as the difference between the mean value of the estimates from the repeated maneuvers, minus the known true value of the parameter. This removes the random variation in the parameter estimates. Results were based on estimated parameters from an ensemble of 20 repeated maneuvers.

All added noise was zero-mean Gaussian, with amplitudes typical of flight test data. In general, when the output noise levels (i.e., noise levels on $C_{Z}$ and $C_{m}$ for the equation-error methods, or on $\alpha, q$, and $a_{z}$ for output-error) were increased, there was an increased variability in the parameter estimates, but no effect on the biases. As expected, using least squares with noise-free regressors resulted in unbiased parameter estimates, whereas least squares with noisy regressors produced biased parameter estimates. If the analysis was done in the frequency domain, or the regressors were smoothed in the time domain first, the unbiased character of the parameter estimates was recovered, but the variability in the parameter estimates increased compared to least squares with noise-free regressors. The output-error method showed slight biases, but low variability in the parameter estimates for different noise sequences. In general, there was good agreement between the results from the output-error method and all of the equation-error methods, except for least squares with noisy regressors. Increased noise level on the regressors caused increased bias in the estimated parameters from the least squares using noisy regressors, but the other two equation-error methods still gave unbiased estimates. Regressor smoothing in the time domain and frequency- 
domain analysis were almost equally effective, but the frequency domain method showed slightly better performance and is computationally more efficient. However, the frequency-domain method does not provide bias term estimates, because the data must be detrended prior to applying the Fourier transform.
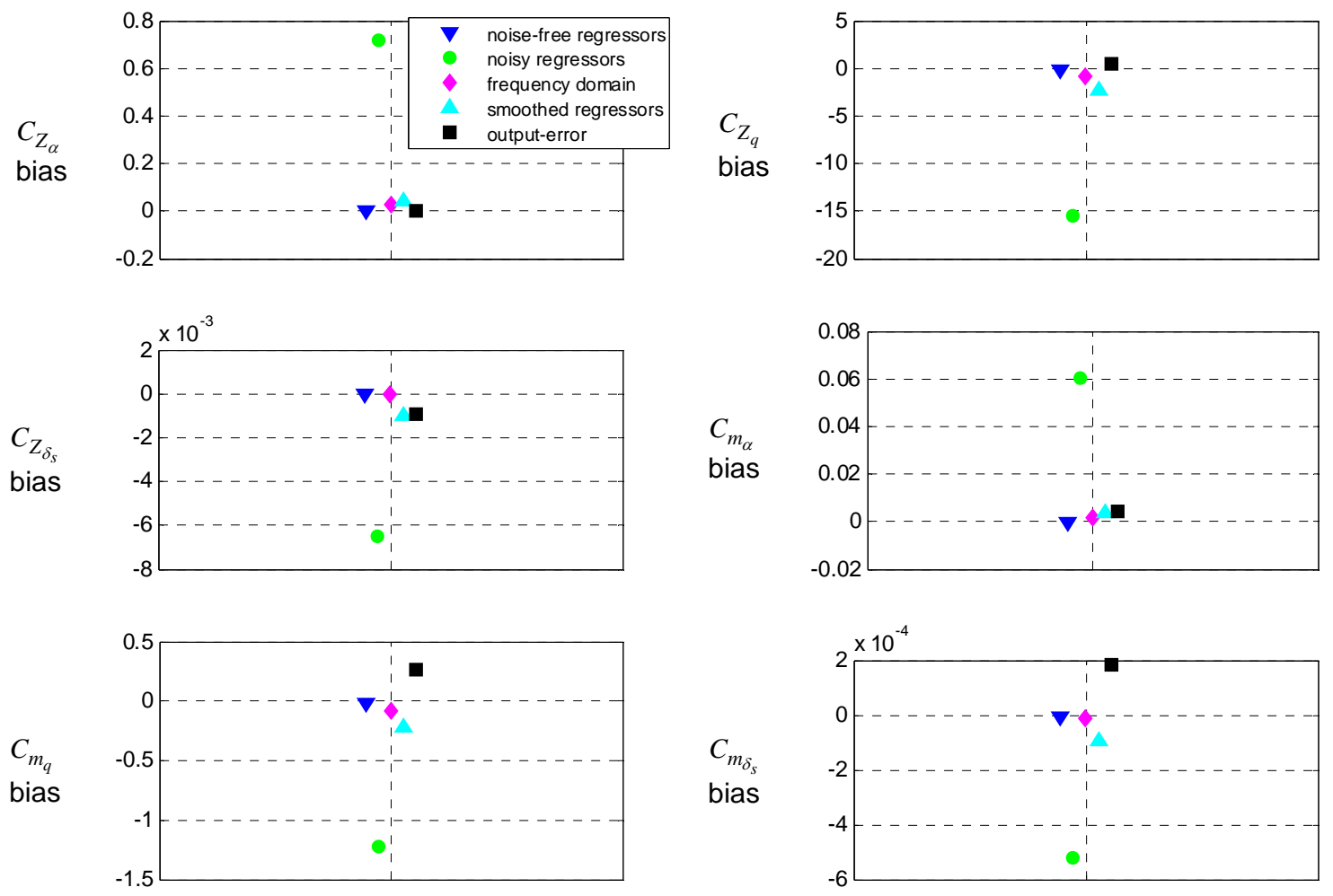

Figure 3. Regressor noise effects on estimated parameter bias

The frequency-domain method and the smoothing method essentially repair the bias in the estimated parameters seen when using least squares with noisy regressors. Both achieve this by smoothing the regressors, since the frequency-domain method implements smoothing by transforming the data for a limited frequency band that includes frequencies of interest, and the smoothing method does something similar by removing high-frequency noise from the time-domain regressors.

\section{Output Matching using Equation-Error Parameter Estimates}

As mentioned earlier, the equation-error method finds parameter estimates that optimize the model match to measured derivative data, roughly speaking. However, there is always a desire to know how well a model matches measured responses from the aircraft, because they are the most physically meaningful. To make this evaluation using equation-error parameter estimates, the meaning of biases must be clearly understood.

For example, in Eq. (8), if the expansion shown is interpreted as a truncated Taylor series model for the pitching moment coefficient, then the modeling functions or regressors should be made from perturbation quantities. If instead the directly measured physical quantities are used for the regressors, Eq. (8) becomes

$$
C_{m}=C_{m_{\alpha}}\left(\alpha_{o}+\Delta \alpha\right)+C_{m_{q}} \frac{\bar{c}}{2 V_{o}}\left(q_{o}+\Delta q\right)+C_{m_{\delta_{s}}}\left(\delta_{s_{o}}+\Delta \delta_{s}\right)+C_{m_{o}}
$$

where the $\Delta$ quantities are perturbations from the reference values with subscript $o$. The reference values are typically from initial trim conditions. Re-arranging, 


$$
C_{m}=C_{m_{\alpha}} \Delta \alpha+C_{m_{q}} \frac{\bar{c}}{2 V_{o}} \Delta q+C_{m_{\delta_{s}}} \Delta \delta_{s}+\left(C_{m_{o}}+C_{m_{\alpha}} \alpha_{o}+C_{m_{q}} \frac{\bar{c}}{2 V_{o}} q_{o}+C_{m_{\delta_{s}}} \delta_{s_{o}}\right)
$$

The bias term in Eq. (36) includes both $C_{m_{o}}$ and terms related to the steady parts of the regressors,

$C_{m_{\alpha}} \alpha_{o}+C_{m_{q}} \frac{\bar{c}}{2 V_{o}} q_{o}+C_{m_{\delta_{s}}} \delta_{s_{o}}$. Consequently, the bias term depends on whether the regressors are assembled

directly from the measured data or from perturbation data where reference values are removed first. The other model parameters (stability and control derivatives) are estimated just the same, regardless of whether directly measured quantities or perturbation quantities are used. This issue does not occur in cases when the reference values are zero for the states and controls used to assemble the regressors, which is common for lateral cases.

When equation-error parameter estimates are used in the equations of motion to compute aircraft responses, any mismatch of the equation-error model to the (derivative) data will be integrated over time. Unless the modeling errors from the equation-error parameter estimation are low-amplitude and zero-mean, the result can be a significant mismatch between measured aircraft outputs and the model outputs computed from the equations of motion using equation-error parameters. In the output-error case, the mismatch between measured outputs and model outputs is intentionally minimized by adjusting the parameter estimates. Consequently, aircraft model responses computed using equation-error estimates will never show as good a match to the measured outputs as output-error parameter estimates. Similarly, using output-error parameter estimates to compute force and moment coefficients will produce inferior fits to the data, compared with using equation-error parameter estimates, which are chosen specifically to optimize the model fit to force and moment coefficient data.

Often, analysts will use equation-error estimates to compute model outputs by fixing the equation-error parameter estimates and letting the bias terms be estimated using output-error parameter estimation. This essentially takes any modeling error that occurs in the equation-error model and assigns it to the bias term. In general, this does not work well, and the resulting model fits to measured outputs are poor. However, it is incorrect to indict the equation-error parameter estimates for this, because they are being used out of context. It is more meaningful to compare the equation-error parameter estimates to output-error parameter estimates, accounting for error bounds and any a priori information, and make judgments of the parameter estimate quality based on that information.

A related practical issue occurs when the flight condition drifts slightly from the beginning to the end of the maneuver. This affects longitudinal cases where the short-period approximation is being used for the data analysis and modeling. The short-period approximation assumes a constant airspeed, but often the airspeed is not constant, and this can show up roughly as a trend in the regressors, such as angle of attack and stabilator deflection. The pitch rate can be approximately zero at both the beginning and end of a maneuver, whereas the angle of attack and stabilator deflection might be different at the beginning and end of the maneuver, due to flight condition drift and the associated airspeed change. The equation-error model for pitching moment coefficient will then exhibit a trend over time, as a result of including regressors (e.g., angle of attack and stabilator deflection) which also have trends over time, due to the flight condition drift. The parameter estimation algorithm tries to account for the flight condition drift in the regressors by adjusting the bias term. This causes a visible mismatch with opposite signs at the beginning and end of the maneuver, as shown in Fig. 4. The solution to this problem is to include an airspeed term in the regression model, then proceed with the parameter estimation as usual. This is equivalent to removing the flight condition drift, or equivalently, applying a time-varying bias corresponding to the airspeed change. In the frequency domain, this 
problem is avoided, because any flight condition drift is removed when the data are detrended prior to Fourier transformation.

\section{E. Estimated Parameter Errors}

Because of model simplifications (e.g., linearizing the nonlinear equations of motion) and aerodynamic model structure inaccuracies (e.g., approximating the real nonlinear dependencies with tractable mathematical functions of instantaneous states and controls), most practical applications of aircraft parameter estimation produce residual sequences that resemble colored noise. Typically, a significant portion of the noise power lies in the same frequency band as the rigid-body dynamics. A method that corrects for this, giving accurate parameter error bounds for time-domain analysis is described in Refs. 5 and 11 and outlined here. The method accounts for colored noise by modifying the calculation of the covariance matrix in Eqs. (15) and (16).

The parameter covariance matrix for colored noise is derived by substituting for $\hat{\boldsymbol{\theta}}$ from Eq. (14) into Eq. (15), and using Eq. (12),

$$
\operatorname{Cov}(\hat{\boldsymbol{\theta}})=E\left[(\hat{\boldsymbol{\theta}}-\boldsymbol{\theta})(\hat{\boldsymbol{\theta}}-\boldsymbol{\theta})^{T}\right]=\left(\boldsymbol{X}^{T} \boldsymbol{X}\right)^{-1} \boldsymbol{X}^{T} E\left(\boldsymbol{v} \boldsymbol{v}^{T}\right) \boldsymbol{X}\left(\boldsymbol{X}^{T} \boldsymbol{X}\right)^{-1}
$$

When $\boldsymbol{v}$ is a zero-mean, weakly stationary random process,

$$
E\left(v v^{T}\right)=E\left[\begin{array}{ll}
v_{i} & v_{j}
\end{array}\right]=\mathcal{R}_{v v}(i-j)=\mathcal{R}_{v v}(j-i) \quad i, j=1,2, \ldots, N
$$

where $\mathcal{R}_{v v}(i-j)$ is the autocorrelation matrix for the residuals. The estimated parameter covariance matrix for correlated residuals can be computed by substituting for $E\left(v v^{T}\right)$ from Eq. (38) into Eq. (37), using an estimate of $\mathcal{R}_{v V}(i-j)$ computed from

$$
\hat{\mathcal{R}}_{v V}(k)=\frac{1}{N} \sum_{i=1}^{N-k} v_{i} v_{i+k}=\hat{\mathcal{R}}_{v V}(-k) \quad k=0,1,2, \ldots, r
$$

where $v_{i}$ is the $i^{\text {th }}$ residual. The index $k$ represents the time separation of the residuals in the summation, and $r$ is the maximum time index difference. Since only proximate residuals are significantly correlated, the value of $r$ can be relatively small, which reduces the required computations. Combining the last three equations,

$$
\operatorname{Cov}(\hat{\boldsymbol{\theta}})=\left(\boldsymbol{X}^{T} \boldsymbol{X}\right)^{-1}\left[\sum_{i=1}^{N} \boldsymbol{x}_{i} \sum_{j=1}^{N} \hat{\mathcal{R}}_{v v}(i-j) \boldsymbol{x}_{j}^{T}\right]\left(\boldsymbol{X}^{T} \boldsymbol{X}\right)^{-1}
$$

where $\boldsymbol{x}_{j}^{T}$ is the $j^{\text {th }}$ row of the $\boldsymbol{X}$ matrix, containing the measured regressors at the $j^{\text {th }}$ data point, and $\hat{\mathcal{R}}_{v v}(i-j)$ is replaced by its estimate from Eq. (39). Note that if the residuals are uncorrelated, then

$$
E\left(v \boldsymbol{v}^{T}\right)=\hat{\mathcal{R}}_{v v}(0) \boldsymbol{I} \approx \hat{\sigma}^{2} \boldsymbol{I}
$$

and Eq. (40) reduces to Eq. (15).

Eqs. (40) with (39) represent a post-processing of the residuals from a least-squares solution to account for the effect of correlated residuals on the parameter covariance matrix. The standard errors for the estimated parameters, corrected for correlated residuals, are found as the square root of the diagonal elements of the covariance matrix calculated from Eq. (40). 
Figure 5 shows a typical colored noise sequence, which is the residual sequence from modeling the pitching moment coefficient for a doublet sequence using the nonlinear F-16 simulation with added white Gaussian noise. Even in the time-domain plot of Fig. 5, it is easy to distinguish prominent low-frequency components, which are attributed to deterministic modeling error. The approach described here assumes that the residual is composed of only random components, but the low-frequency components are serially correlated in time, resulting in colored noise. This does not correspond exactly to the physical situation, because the low-frequency components of the residual are really from deterministic modeling errors. However, the shift in how the residual is viewed makes it possible to compute accurate error bounds for the estimated parameters. In effect, the

problem is changed to the following: given that the true model structure is the one postulated, and the noise is correlated in time, what are the errors in the parameters? The fact that the model structure is not exactly correct is manifested by colored noise and increased error bounds for the parameters in the specified model structure.

In the frequency domain, the residuals are no longer serially correlated, because each data point in the regressor matrix has changed from measurements at a single sampling time to Fourier coefficients for a single frequency. So,

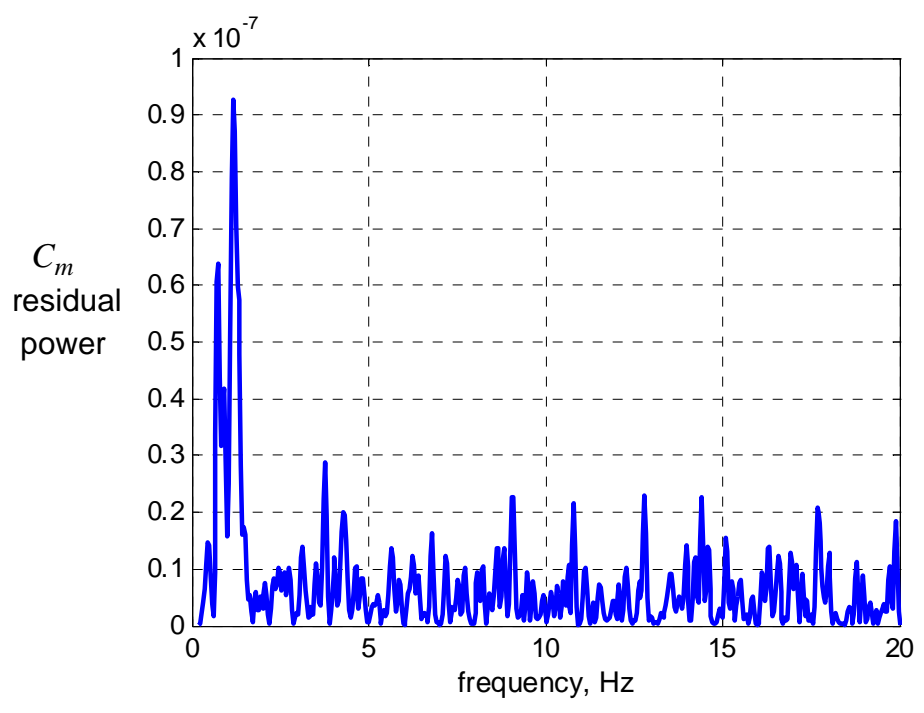

Figure 6. Pitching moment residual power spectral density the assumption of uncorrelated residuals is acceptable in the frequency domain, but the assumption that the noise variance magnitude is constant over all frequencies (cf. Eq. (21)) is not. Figure 6 shows that the power spectral density of the model residuals in Fig. 5 does not have constant magnitude. However, if the frequency-domain analysis is limited to the frequency band containing the rigid-body frequencies, which is where the large components appear in Fig. 6, then the residuals can be approximated as having constant magnitude. In that case, the theory matches the application, and the estimated parameter error bounds are calculated accurately without any corrections. As a consequence, if equation-error is applied in the frequency domain with the transform frequencies chosen to approximately correspond to the large magnitude components of the residuals in the frequency domain, then standard calculations outlined above in Eqs. (20)-(22) can be used without modification to calculate accurate standard errors for the estimated parameters.

Computed error bounds are accurate using the frequency-domain method or using the colored noise calculation with the regressors smoothed in the time domain. If the regressors are noisy and not smoothed, then using the colored noise correction will not give accurate error bounds. 


\section{F. Multiple Maneuvers}

Often there is a desire to estimate aerodynamic model parameters using data from more than one flight test maneuver. This is useful when individual maneuvers have good information content for estimating some parameters but not others. Sometimes multiple maneuvers taken together can provide more complete information for parameter estimation. Equation-error allows concatenation of the data from various maneuver by simply stacking the data together in the measured output vector $\boldsymbol{z}$, and the regressor matrix $\boldsymbol{X}$ in Eq. (12). The implicit assumptions made in doing this, in addition to the usual equation-error assumptions, are: 1 ) the noise $\boldsymbol{v}$ is of the same character for all concatenated maneuvers, and 2) the experimental conditions of the concatenated maneuvers are sufficiently similar that the model parameters can be expected to be the same. Violating the first assumption leads to inaccuracies in the estimated parameter uncertainties. If the second assumption is violated, the parameter estimation results are some weighted average of the results that would have been obtained from each of the concatenated maneuvers analyzed individually. Parameter estimates from the equation-error method are independent of the ordering of the data points, so it does not matter how the data points (i.e., the rows) are ordered in $\boldsymbol{z}$ and $\boldsymbol{X}$ as far as the parameter estimates are concerned; however, the ordering is important for accurately computing parameter standard errors, as discussed in the previous section.

An equivalent method for analyzing multiple maneuvers is to use a priori parameter estimates in the equation-error formulation. If the data from two maneuvers are designated by subscripts 1 and 2 , and the data from both maneuvers are analyzed together, then Eq. (12) becomes

$$
\left[\begin{array}{l}
\boldsymbol{z}_{1} \\
\boldsymbol{z}_{2}
\end{array}\right]=\left[\begin{array}{l}
\boldsymbol{X}_{1} \\
\boldsymbol{X}_{2}
\end{array}\right] \theta+\left[\begin{array}{l}
\boldsymbol{v}_{1} \\
\boldsymbol{v}_{2}
\end{array}\right]
$$

and the analysis proceeds as usual, assuming that the noise sequences $\boldsymbol{v}_{1}$ and $\boldsymbol{v}_{2}$ are similar, and the matrices $\boldsymbol{X}_{1}$ and $\boldsymbol{X}_{2}$ contain analogous columns of regressors of the same form, implementing a common model structure for both maneuvers. If instead the analysis of the data from maneuver 1 was done previously, the cost function to be minimized for maneuver 2 can include a priori information about the parameters from the maneuver 1 results, as follows

$$
J(\theta)=\frac{1}{2 \sigma_{2}^{2}}\left(z_{2}-X_{2} \theta\right)^{T}\left(z_{2}-X_{2} \theta\right)+\frac{1}{2}\left(\theta-\theta_{1}\right)^{T} \Sigma_{1}^{-1}\left(\theta-\theta_{1}\right)
$$

where

$$
\hat{\boldsymbol{\theta}}_{1}=\left(\boldsymbol{X}_{1}^{T} \boldsymbol{X}_{1}\right)^{-1} \boldsymbol{X}_{1}^{T} \boldsymbol{z}_{1} \quad \operatorname{Cov}\left(\hat{\boldsymbol{\theta}}_{1}\right)=\sigma_{1}^{2}\left(\boldsymbol{X}_{1}^{T} \boldsymbol{X}_{1}\right)^{-1} \equiv \boldsymbol{\Sigma}_{1}
$$

The parameter estimation results in this case are obtained from ${ }^{5}$

$$
\hat{\boldsymbol{\theta}}=\left[\left(\boldsymbol{X}_{2}^{T} \boldsymbol{X}_{2}\right) / \sigma_{2}^{2}+\Sigma_{1}^{-1}\right]^{-1}\left[\left(\boldsymbol{X}_{2}^{T} \boldsymbol{z}_{2}\right) / \sigma_{2}^{2}+\boldsymbol{\Sigma}_{1}^{-1} \boldsymbol{\theta}_{1}\right]
$$

Substituting from Eq. (44),

$$
\hat{\boldsymbol{\theta}}=\left[\left(\boldsymbol{X}_{2}^{T} \boldsymbol{X}_{2}\right) / \sigma_{2}^{2}+\left(\boldsymbol{X}_{1}^{T} \boldsymbol{X}_{1}\right) / \sigma_{1}^{2}\right]^{-1}\left[\left(\boldsymbol{X}_{2}^{T} \boldsymbol{z}_{2}\right) / \sigma_{2}^{2}+\left(\boldsymbol{X}_{1}^{T} \boldsymbol{z}_{1}\right) / \sigma_{1}^{2}\right]
$$

Assuming the noise sequences $\boldsymbol{v}_{1}$ and $\boldsymbol{v}_{2}$ are similar, $\sigma_{1}^{2} \approx \sigma_{2}^{2} \equiv \sigma^{2}$, so that

$$
\hat{\boldsymbol{\theta}}=\left[\boldsymbol{X}_{2}^{T} \boldsymbol{X}_{2}+\boldsymbol{X}_{1}^{T} \boldsymbol{X}_{1}\right]^{-1}\left[\boldsymbol{X}_{2}^{T} \boldsymbol{z}_{2}+\boldsymbol{X}_{1}^{T} \boldsymbol{z}_{1}\right]
$$


which is the same estimate that would be obtained by concatenating the data. Similarly, the covariance matrix associated with the parameter estimates in Eq. (45) is

$$
\operatorname{Cov}(\hat{\boldsymbol{\theta}})=\left[\left(\boldsymbol{X}_{2}^{T} \boldsymbol{X}_{2}\right) / \sigma_{2}^{2}+\Sigma_{1}^{-1}\right]^{-1}=\left[\left(\boldsymbol{X}_{2}^{T} \boldsymbol{X}_{2}\right) / \sigma_{2}^{2}+\left(\boldsymbol{X}_{1}^{T} \boldsymbol{X}_{1}\right) / \sigma_{1}^{2}\right]^{-1}
$$

or, assuming the noise sequences $\boldsymbol{v}_{1}$ and $\boldsymbol{v}_{2}$ are similar,

$$
\operatorname{Cov}(\hat{\boldsymbol{\theta}})=\sigma^{2}\left[\boldsymbol{X}_{2}^{T} \boldsymbol{X}_{2}+\boldsymbol{X}_{1}^{T} \boldsymbol{X}_{1}\right]^{-1}
$$

where $\sigma^{2}$ is estimated from Eq. (16), based on all of the data from both maneuvers 1 and 2. Again, this expression is the same one that would be obtained by concatenating the data.

This development shows that as long as the assumptions listed above are acceptable, using a priori values for the parameter estimates and covariance matrix is equivalent to concatenating the data from more than one maneuver. The result shown here obviously extends to more than two maneuvers. Using a priori values for parameter estimates and the covariance matrix is much more convenient than concatenating data, especially for many maneuvers. This provides an easy way to incorporate the information from additional maneuvers on parameters already calculated from other data. If the noise sequences for the maneuvers to be combined have different variances, the more general expressions in Eqs. (45) and (48) can be used.

In the frequency domain, if multiple maneuvers are transformed using the same frequencies, then the data for the combination of multiple maneuvers is simply the addition of the frequency-domain data at each corresponding frequency. The analysis then proceeds as usual using Eqs. (19)-(22). This straightforward approach assumes that common transform frequencies can be used for multiple maneuvers, which may have different time lengths. This can be done with high accuracy using the Fourier transform method described in Ref. 5 or 6 .

\section{G. Data Collinearity}

Data collinearity is a linear or nearly-linear relationship among regressors, which simply means that some model terms are nearly the same as a linear combination of other terms in the model. Existence of data collinearity can compromise parameter estimation results, because when regressors are nearly collinear, there is ambiguity in how the variations in the measured output can be modeled. This is true regardless of the parameter estimation method, but the issue is easier to study in detail for the equation-error method.

For the equation-error method, the detrimental effects of data collinearity are closely tied to the noise levels on the measurements. In fact, if the noise levels are zero, data collinearity has no effect at all on least-squares parameter estimates. This was demonstrated using simulated data for longitudinal short period linear dynamics of the F-16.

A doublet sequence was applied to the stabilator, and the simulated aircraft responses were corrupted with white Gaussian noise. A simple pitch rate feedback to the stabilator was used to correlate the stabilator and pitch rate regressors. This situation is a common cause of data collinearity in practice. By adjusting the feedback gain, the regressors could be correlated to an arbitrary extent. Pair-wise regressor correlations were defined by:

$$
\begin{gathered}
r_{j k} \equiv \frac{C_{j k}}{\sqrt{C_{j j} C_{k k}}}=\frac{\operatorname{Cov}\left(\hat{\theta}_{j}, \hat{\theta}_{k}\right)}{\sqrt{\operatorname{Var}\left(\hat{\theta}_{j}\right) \operatorname{Var}\left(\hat{\theta}_{k}\right)}} \quad j, k=1,2, \ldots, n_{p} \\
-1 \leq r_{j k} \leq 1 \quad j, k=1,2, \ldots, n_{p}
\end{gathered}
$$

With no noise on the measured output $\left(C_{Z}\right.$ or $C_{m}$ ) and using noise-free regressors, the ordinary least squares solution given in Eqs. (14)-(16) very accurately computed the true parameter values, even when the maximum 
absolute value of the regressor correlation exceeded 0.99. The solid line in Fig. 7 shows this result for the $C_{m_{\alpha}}$ parameter.

This demonstrates that extremely high data collinearity can be handled using the default double-precision arithmetic in MATLAB ${ }^{\circledR}$, as long as there is no noise. The problems occur when measurement noise is added. Keeping the regressors noise-free and adding noise to the measured output produces approximately unbiased estimates, as shown in Fig. 7. However, when noise is added to the regressors, the estimated parameter bias is significant. If least squares in the frequency domain is applied to the case where both the regressors and the measured output are noisy, nearly unbiased parameter estimates are recovered, and the parameter estimates are as good as when the regressors had no noise at all. This result comes from using a limited frequency band for the Fourier transform, as discussed earlier, which effectively removes the wide-band noise from the regressors in the frequency domain. Similar results were found for the other model parameters, and for inputs different than a doublet.

In Fig. 7, note that when the maximum regressor correlation exceeds approximately 0.9 , the results degrade in all cases. Consequently, a good practical rule of thumb is that the effects of

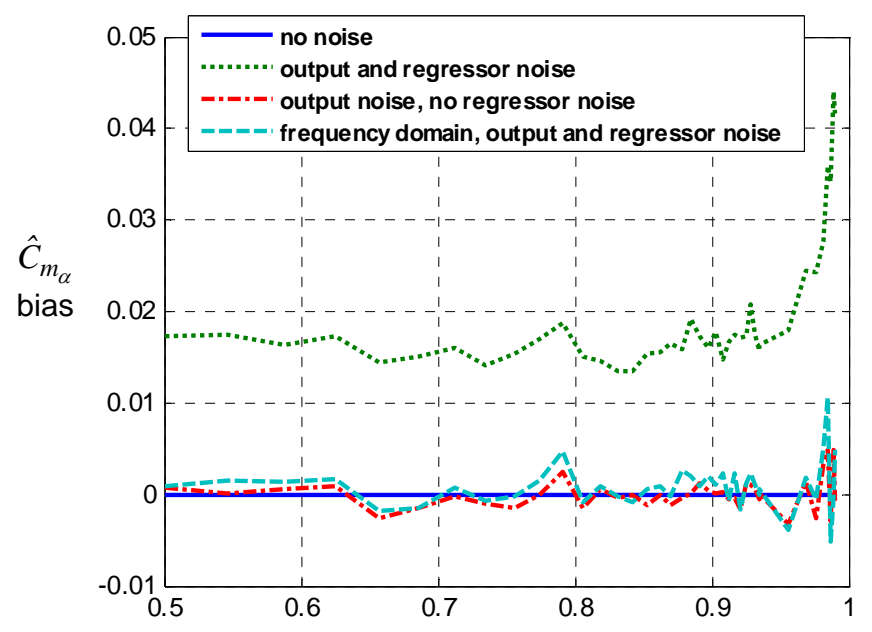

Figure 7. Data collinearity and noise effects on estimated parameter bias data collinearity get worse when the absolute value of regressor correlation exceeds 0.9. Increased noise levels show the same trends, but the bias in the estimated parameters increases for least squares with noisy regressors. Increased noise on the outputs does not affect the bias, but increases the random variation in the parameter estimates in all cases. This empirical finding is consistent with the expression derived for the bias in Eq. (32).

In summary, noise on the regressors combined with data collinearity will produce significant biases in estimated parameters using least squares in the time domain. Measurement noise must be present as a catalyst for the data collinearity to cause inaccuracies in the parameter estimates. If the regressors and outputs are noise-free, least squares using ordinary double-precision arithmetic can accurately estimate model parameters for pair-wise regressor correlations as high as 0.99; however, when noise is added to the regressors, the estimated parameters are biased. Using least squares in the frequency domain repairs the adverse effects of data collinearity by effectively removing the wide-band noise on the regressors.

\section{Conclusions}

Several practical issues related to applying the equation-error method to aircraft parameter estimation problems were examined and discussed. The issues included numerical differentiation of noisy data, maximum likelihood parameter estimation, biases in estimated parameters, computing aircraft responses using parameters estimated with equation-error methods, calculating estimated parameter errors, parameter estimation based on data from more than one maneuver, and data collinearity. Some limitations and deficiencies of the equation-error method were shown to be removed by advances in applying the technique. Specifically, applying the equation-error method in the frequency domain was shown to have advantages in computational efficiency, accurate computation of the estimated parameter errors, minimizing biases in the estimated parameters, and robustness to the adverse effects of data collinearity. The equation-error method is applicable to aircraft modeling problems that are increasingly prevalent in modern aircraft, such as nonlinear aerodynamic modeling, large data sets, and aircraft that are open-loop unstable.

Practical issues were examined using data from an F-16 nonlinear simulation, with realistic colored noise sequences added to the computed aircraft responses. This allowed a clear view of the effectiveness of various 
approaches to modeling problems, because the true values of the model parameters were known. This approach is necessary to fully understand and validate tools for flight data analysis and modeling.

Software tools for all the techniques and investigations included here, along with many other methods used in aircraft system identification, can be found in the software package called SIDPAC (ystem IDentification Programs for AirCraft), which is available with Ref. 5 .

The venerable equation-error method, implemented using modern analytical approaches and numerical techniques, was shown to be accurate and useful for aircraft parameter estimation problems.

\section{References}

${ }^{1}$ Greenberg, H. "A Survey of Methods For Determining Stability Parameters of an Airplane From Dynamic Flight Measurements,” NACA TN 2340, April 1951.

${ }^{2}$ Maine, R.E. and Iliff, K.W. "Application of Parameter Estimation to Aircraft Stability and Control, The Output Error Approach,” NASA RP 1168, June 1986.

${ }^{3}$ Garza, F.R. and Morelli, E.A. “A Collection of Nonlinear Aircraft Simulations in MATLAB ${ }^{\circledR}$,"

NASA / TM-2003-212145, 2003.

${ }^{4}$ Getting Started with MATLAB, Version 7, The MathWorks, Natick, MA, 2005

${ }^{5}$ Klein, V. and Morelli, E.A. Aircraft System Identification - Theory and Practice, AIAA Education Series, AIAA, Reston, VA, 2006.

${ }^{6}$ Morelli, E.A. "High Accuracy Evaluation of the Finite Fourier Transform using Sampled Data," NASA TM 110340, 1997.

${ }^{7}$ Wood, G.A. "Data Smoothing and Differentiation Procedures in Biomechanics," Exercise and Sport Science Reviews, Vol. 10, pp. 308-362.

${ }^{8}$ Lanczos, C. Applied Analysis, Dover Publications, New York, NY, 1988.

${ }^{9}$ Morelli, E.A. "Estimating Noise Characteristics from Flight Test Data using Optimal Fourier Smoothing," Journal of Aircraft, Vol. 32, No. 4, 1995, pp. 689-695.

${ }^{10}$ Bendat, J.S. and Piersol, A.G. Random Data Analysis and Measurement Procedures, 2nd Ed., John Wiley \& Sons, New York, NY, 1986.

${ }^{11}$ Morelli, E.A. and Klein, V. “Accuracy of Aerodynamic Model Parameters Estimated from Flight Test Data," Journal of Guidance, Control, and Dynamics, Vol. 20, No. 1, 1997, pp. 74-80.

Table 1. Geometry and mass properties of the F-16 aircraft

\begin{tabular}{cc}
\hline \hline length $\bar{c}, \mathrm{ft}$ & 11.32 \\
\hline wing span $b, \mathrm{ft}$ & 30 \\
\hline wing area $S, \mathrm{ft}^{2}$ & 300 \\
\hline$x_{\text {ref }}, \mathrm{ft}$ & $0.35 \bar{c}$ \\
\hline$y_{\text {ref }}, \mathrm{ft}$ & 0.000 \\
\hline$z_{r e f}, \mathrm{ft}$ & 0.000 \\
\hline$x_{c g}, \mathrm{ft}$ & $0.25 \bar{c}$ \\
\hline$y_{c g}, \mathrm{ft}$ & 0.0 \\
\hline$z_{c g}, \mathrm{ft}$ & 0.0 \\
\hline$m$, slugs & 647.2 \\
\hline$I_{x}$, slugs- $\mathrm{ft}^{2}$ & 9,496 \\
\hline$I_{y}$, slugs- $\mathrm{ft}^{2}$ & 55,814 \\
\hline$I_{z}$, slugs- $\mathrm{ft}^{2}$ & 63,100 \\
\hline$I_{x z}$, slugs- $\mathrm{ft}^{2}$ & 982 \\
\hline \hline
\end{tabular}

